\title{
Switching serotonin reuptake inhibitors may be of benefit in people with obsessive compulsive disorder
}

Denys D, van Megen HJ, van der WN, et al. A double-blind switch study of paroxetine and venlafaxine in obsessive-compulsive disorder. J Clin Psychiatry 2004;65:37-43.

\section{What is the efficacy of switching serotonin reuptake inhibitors (SRI) in people with obsessive compulsive disorder (OCD) who have not responded to the first SRI?}

\section{METHODS}

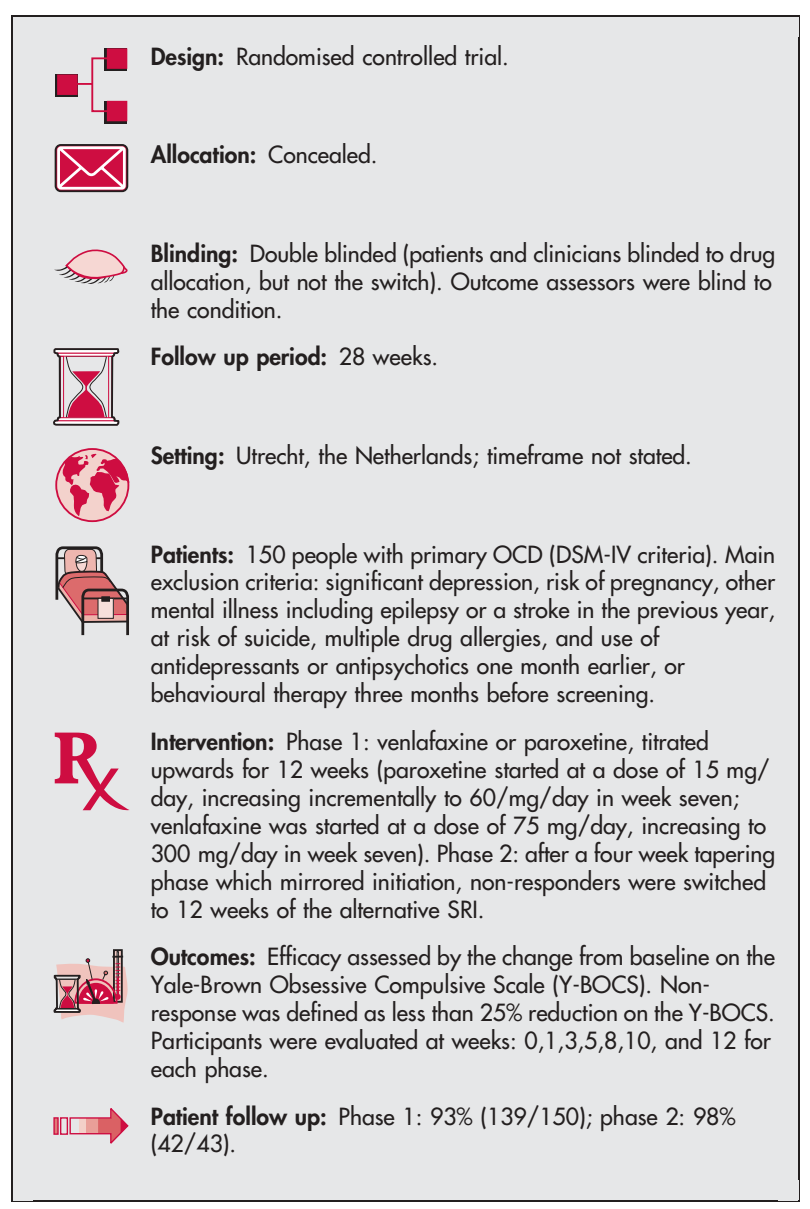

\section{MAIN RESULTS}

Phase 1: 88 participants were rated as responders $(63 \%)$ and 51 as non-responders (37\%). $43 / 51$ were enrolled in the second phase (16 to venlafaxine and 27 to paroxetine). Phase 2: 18 (42\%) of the 43 participants in phase 2 benefited from the switch to the other SRI. After 12 weeks, responder rates were 56\% (15/27) for paroxetine and $19 \%(3 / 16)$ for venlafaxine. Y-BOCS score reduced from baseline in both groups, but there was no significant difference in score reduction between groups (6.5 (SD 7.1) in the paroxetine group $v$ For correspondence: Damiaan Denys, UMC, 3508 GA Utrecht, the Netherlands; D.A.J.P.denys@azu.nl

Sources of funding: Wyeth, Hoofddorp, the Netherlands, and Glaxo SmithKline, Zeist, the Netherlands.
1.8 (SD 3.5) in the venlafaxine group; $\mathrm{p}=0.13$ ). However, paroxetine significantly improved response rate compared with venlafaxine $(66 / 102(65 \%)$ with paroxetine $v 39 / 91$ (43\%) with venlafaxine; $\mathrm{p}<0.002)$.

\section{CONCLUSIONS}

In people with refractory OCD, switching SRIs may be of benefit.

\section{NOTES}

Authors note that although they have assumed that improvements in phase 2 are due to switching SRIs, any benefits may result from continuing treatment for 28 weeks.

\section{Commentary}

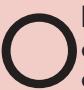
bsessive compulsive disorder (OCD) is a chronic and often disabling condition with marked social and occupational disabilities as well as a poor quality of life. Optimal treatment involves both pharmacological and cognitive behavioural techniques. Refractory OCD may respond to SSRI switching to a different antidepressant. ${ }^{1}$ The efficacy and effectiveness of such switching has not been thoroughly researched. Denys et al report that after two consecutive SRI trials, $73 \%$ of participants responded to treatment.

The patient group was select in that they had pure OCD and were referrals to a specialist academic centre. The criteria excluding people with any comorbid disorders or recent therapy mean that findings from this trial are likely to be evidence for efficacy rather than the clinical effectiveness in real service settings. The data do support switching to be an effective strategy. However, the authors take the subanalyses, which appear to be post hoc, a little too far in trying to promote paroxetine as being "superior" to venlafaxine. It is impossible to totally exclude carry over effects, even after a four week washout period. Furthermore, the study did not report dosages, or account for them in the analyses. Higher doses may be more effective but venlafaxine at low dose acts mostly as an SRI agent rather than a noradranergic agent. ${ }^{1}$

Overall, $67 \%$ of patients responded to paroxetine compared with $44 \%$ to venlafaxine. Putting aside the carry over effect issue, this suggests paroxetine is more effective in pure OCD due to its SSRI properties. This is not an unexpected finding as venlafaxine is thought to act through noradranergic mechanisms and people with affective or other anxiety symptoms were likely to have been excluded from the study. This study provides good evidence that switching is a useful strategy to deploy for refractory treatment of pure $O C D$ and that overall, paroxetine may be more effective in the absence of comorbid disorders or complications. The trial did not assess past use of CBT or treatment histories. These in themselves may affect response to interventions at a later date.

In terms of clinical practice, the study should support clinicians to use switching for pure refractory $O C D$, although studies examining more comorbidity and complex mental states are required before recommending the routine practice of switching in OCD.

Kamaldeep Bhui BSc, MBBS, MRCPsych, MSc, MD Barts and the London, Queen Mary, University of London, UK

1 Kaplan A, Hollander E. A review of pharmacologic treatments for obsessive- compulsive disorder. Psychiatr Serv 54:1111-18.

2 Hollander E, Kahn J. Review: in obsessive-compulsive disorder, clomipramine may be more effective than selective serotonin reuptake inhibitors after controlling for other factors. EBMH 2003;6:23. 